\title{
Optimal Frequency Regulation V2G Control with DOD of EV Battery
}

\author{
Seungmin Jung, Minhan Yoon, Jaewan Suh
}

\begin{abstract}
As the proportion of electric vehicles increases, interest in Vehicle to Grid (V2G) service is increasing. Many studies are underway to use V2G for peak shaving and frequency regulation in power system. However, V2G can shorten battery cycle life for electric vehicle $(\mathrm{EV})$ which is the most variable part in EV. Hence battery cycle life should be considered in V2G service. As well as the number of discharges, depth of discharge (DOD) also highly affects battery cycle life. High depth of discharge reduces the cycle life of the $E V$ battery exponentially. However, conventional droop control, which has been used for frequency regulation, controls the active power linearly without regard to the DOD. This paper proposes an optimal frequency regulation V2G control which considers the DOD of $E V$. Proposed method uniformly distributes the discharge for V2G. Therefore battery cycle life is preserved and inconvenience of $E V$ owner from discharge is reduced. The case study result demonstrates the advantages of the proposed method over the conventional droop method. Battery cycle life of entire EV is preserved and energy consumption under V2G is uniformly distributed.
\end{abstract}

Keywords : Cycle life of battery, Droop control, Electric vehicle, Frequency regulation, Vehicle to Grid

\section{INTRODUCTION}

Worldwide sales of electric vehicles in 2017 increased by 54\% from 2016 to over 1 million units[1]. It is expected to operate 18.7 million vehicles, which is $7 \%$ of all US vehicles by 2030, and 9.6 million chargers will be needed[2]. Large penetration of EV in power system can cause many problems such as imbalance between electric demand and supply[3,4]. And power quality and voltage quality problems can arise due to EV chargers in distribution system[5-8]. As the penetration rate of electric vehicles and charging stations increases, interest in V2G is also increasing to solve the challenges due to large EV penetration. V2G means a service that supplies electric power to the power system through charging and discharging of EV[9]. Through V2G, EV can be transformed into controllable resources in power system from operational challenges.

Until recently research has been mainly focused on peak shaving with V2G[10-12]. In peak shaving operation, EVs are charged during off-peak hours and discharged during peak operation costs and necessity of new power plants can be reduced. However, there are several challenges to realize the

\section{Revised Manuscript Received on July 22, 2019.}

First Author Name*, his/her department, Name of the affiliated College or University/Industry, City, Country. Email: xyz1@blueeyesintlligence.org

Second Author Name, department, Name of the affiliated College or University/Industry, City, Country. Email: xyz2@blueeyesintlligence.org

Third Author Name, department, Name of the affiliated College or University/Industry, City, Country. Email: xyz3@blueeyesintlligence.org hours to use as reserve. Through peak shaving, power system

V2G service for peak shaving. First challenge is the difficulty of predicting operation pattern of $\mathrm{EV}$. In order to use $\mathrm{EV}$ in peak shaving, it is necessary to predict the charging pattern, the expected departure time, and the minimum SOC of EV required at the start[13,14]. Second problem is the influence on cycle life of EV battery. Battery is one of the most valuable components of EV and it has relatively short life span compared to other component. Peak shaving from EV needs a lot of energy from the battery which means that the depth of discharge (DOD) gets large. DOD highly affects to battery cycle life and the larger the DOD every cycle means the smaller the available life cycle will be[15, 16]. Recent days, many researches are underway to use V2G for frequency regulation[17, 18]. When $\mathrm{V} 2 \mathrm{G}$ is applied to frequency regulation, prediction is easier than peak shaving and the amount of energy for $\mathrm{V} 2 \mathrm{G}$ is reduced. Since the unit price of frequency regulation reserve is relatively high, power system operation costs can be significantly reduced by V2G. To save the battery life cycle in V2G operation, DOD should be considered for V2G efficiency because DOD highly affects battery life cycle. In this paper, optimal frequency regulation V2G control, which considers the DOD of each EV in V2G service, is proposed. The conventional droop control did not consider the DOD of each EV but the proposed control can save battery cycle life by considering DOD when allocating the discharge. Realistic data from PJM and tesla are used for case study. Case study with MATLAB shows the effectiveness of propose control.

\section{DOD AND CYCLE LIFE OF BATTERY}

Battery cycle life is number of charge and discharge cycles until battery reaches its End of Life (EOL) which is typically defined as $20 \%$ reduction in rated capacity of battery. Cycle life highly depends on the DOD and number of cycles. Charging and discharging in high DOD results stress to battery cell and reduce the cycle life of battery[15]. In 50\% DOD case, the expected number of equivalent full cycles is 2.7 times longer than expected number of equivalent full cycles of $80 \%$ DOD case. It is general for all cell chemistries such as lead acid or lithium-ion battery, more or less. Relation between DOD and cycle life of Li-ion battery is expressed as[15].

$$
\text { Cycle life }=1591.1 \cdot D O D^{-2.089}
$$

However, it is unreasonable to directly compare the life cycles of different DODs, it is necessary to use the equivalent full cycle life which is calculated by multiplying cycle life by DOD. 
Equivalent full cycle life $=1591.1 \cdot D O D^{-1.089}$

Fig.1 shows the relationship between the DOD and equivalent full cycle life. As shown in Fig.1, the DOD and equivalent full cycle life have an inverse proportion. Hence operating the EV battery with low DOD is very crucial to protect the cycle life of battery.

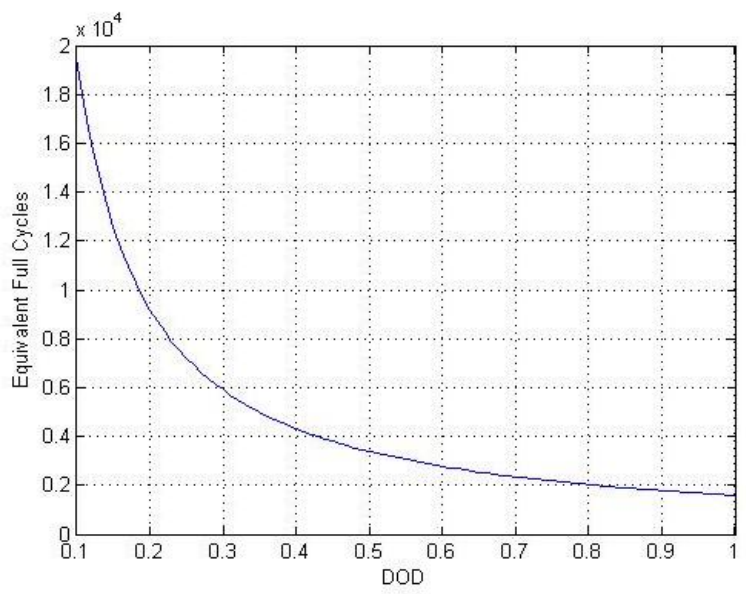

Fig. 1. DOD and equivalent full cycle of life

\section{FREQUENCY REGULATING V2G CONTROL WITH DOD}

Power system frequency is held by frequency regulation reserve which adjust the output quickly[19]. Normally, operating hour of frequency regulation reserve is not long. Therefore, DOD can be kept low in V2G operation and battery cycle life can be protected. In addition, predicting the schedule of $\mathrm{EV}$ is easier because frequency regulation is a short time unit operation. In those reason, frequency regulation reserve is suitable for $\mathrm{V} 2 \mathrm{G}$ application taking account of battery cycle life. The droop control has been used most widely for power system frequency regulation[20].

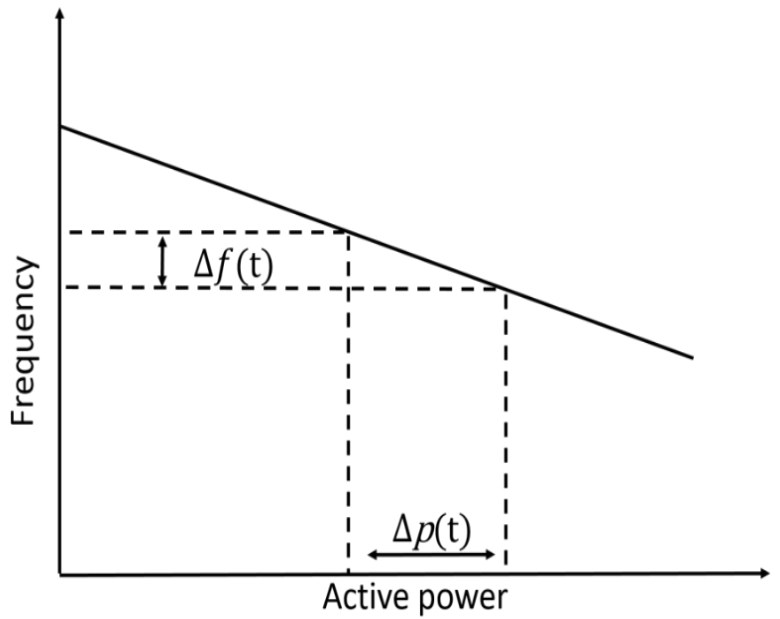

Fig. 2. Droop control in frequency regulation

Droop control has the advantage of being reliable and not requiring additional communication devices. Frequency deviation is used for signal for droop control as shown in the following equation

$$
P-P_{0}=-k\left(f-f_{0}\right)
$$

where $P_{0}$ and $f_{0}$ are base active power and base frequency of the power system. $\mathrm{k}$ is droop control constant for frequency regulation. Fig. 2 shows the droop characteristics between frequency and active power. Since the cycle life of the battery decreases exponentially with increasing DOD, uniform distribution of the DOD is very important. However, conventional droop control does not consider DOD of EV battery because it is proportional control over frequency deviation. Thus, in this study, conventional droop control is implemented to distribute the frequency regulation reserve considering the DOD of the entire EVs battery. In proposed control, droop control constant $\mathrm{k} \_\mathrm{i}$ is calculated as follow

$$
k_{i}(t)=-\frac{\frac{1}{D O D_{i}(t)}}{D O D_{\text {total }}(t)} k_{0}
$$

where $k_{0}$ is initial droop constant of ith $\mathrm{EV}, D O D_{i}$ is DOD of $i$ th $\mathrm{EV}$, and $\mathrm{DOD}_{\text {total }}$ is sum of the DOD inverse as shown below

$$
D O D_{\text {total }}(t)=\sum_{m=1}^{n} \frac{1}{D O D_{m}(t)}
$$

From (3) and (4) implemented droop control which considers the DOD is expressed as follow

$$
P_{i}(t)=k_{i}(t) \Delta f(t)
$$

where $P_{i}$ is charging/discharging power of ith $\mathrm{EV}$ and $\Delta \mathrm{f}$ is frequency deviation. The implemented droop control allocates the frequency regulation reserve in real time, taking into account the DOD of each EV and the overall EV. EV batteries with a relatively low DOD are preferentially used for frequency regulation. And EV batteries with high DOD will be relatively excluded in $\mathrm{V} 2 \mathrm{G}$ frequency regulation.

\section{CASE STUDY}

In this case study, V2G operation of EV for a day is simulated by MATLAB. Historical operation data of PJM on 01. Feb. 2018 is used for 24 hours simulation. Number of EV which participates in V2G service are assumed as 10. Tesla model S 90D and Tesla single charger model data are used for case study. Table I shows the parameters and values for case study. Frequency regulation signal of PJM on 01. Feb. 2018 is shown in Fig.3. From those regulation signals, frequency fluctuation on 01. Feb. 2018 is estimated and used for V2G control signal in case study. As shown in Fig.3, frequency regulation reserves are not fully operated for most of time in real power system operation. Fig. 4 shows the result of case study which compares between conventional droop control and proposed control for 24 hours. Initial DODs at 0 hour are randomly chosen and EVs were assumed to be parked for 24 hours. Energy consumption during frequency regulation in EV batteries is calculated by Fig.3 and (6).

Table- I: Parameters and values for case study

\begin{tabular}{|c|c|}
\hline Parameter & Value \\
\hline Number of EVs & 10 \\
\hline Battery capacity & $90 \mathrm{kWh}$ \\
\hline Power of charger & $10 \mathrm{~kW}$ \\
\hline
\end{tabular}




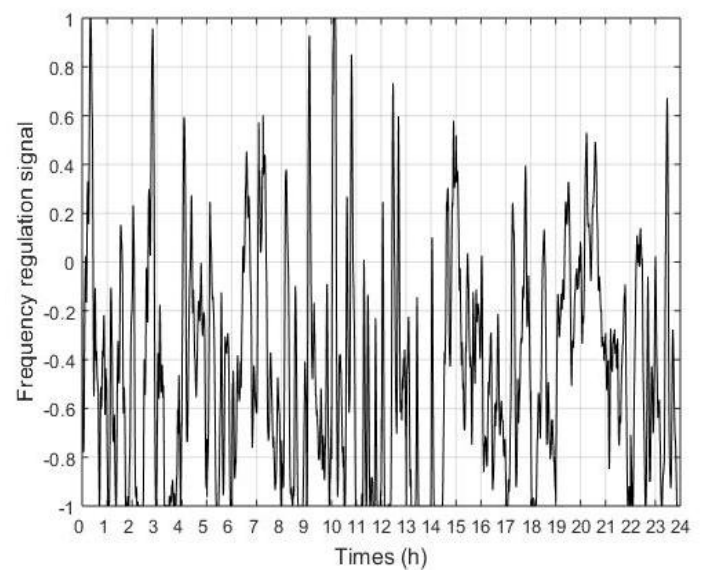

Fig. 3. Frequency regulation signal for 24 hours

\section{RESULT AND DISCUSSION}

By applying the proposed control in $\mathrm{V} 2 \mathrm{G}$, discharges for frequency regulation in EVs are uniformly distributed. From DOD in Fig.4 and (2), total consumed cycle life of EV battery can be calculated.

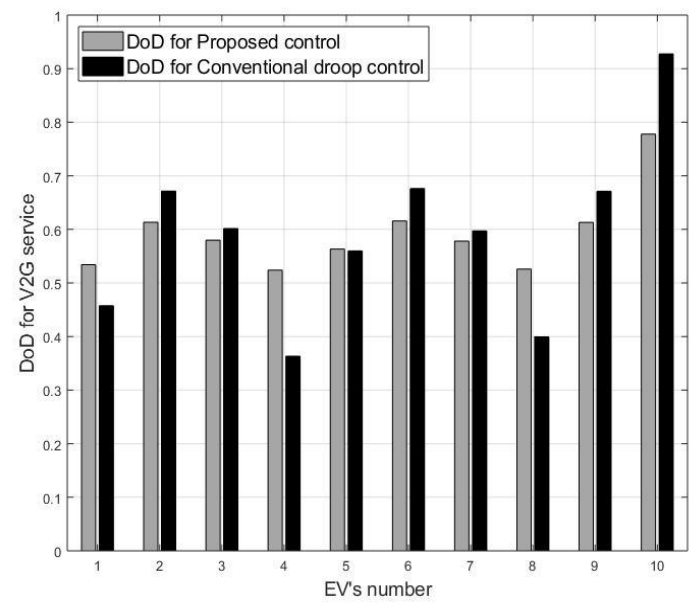

Fig. 4. Comparison between conventional droop and proposed control

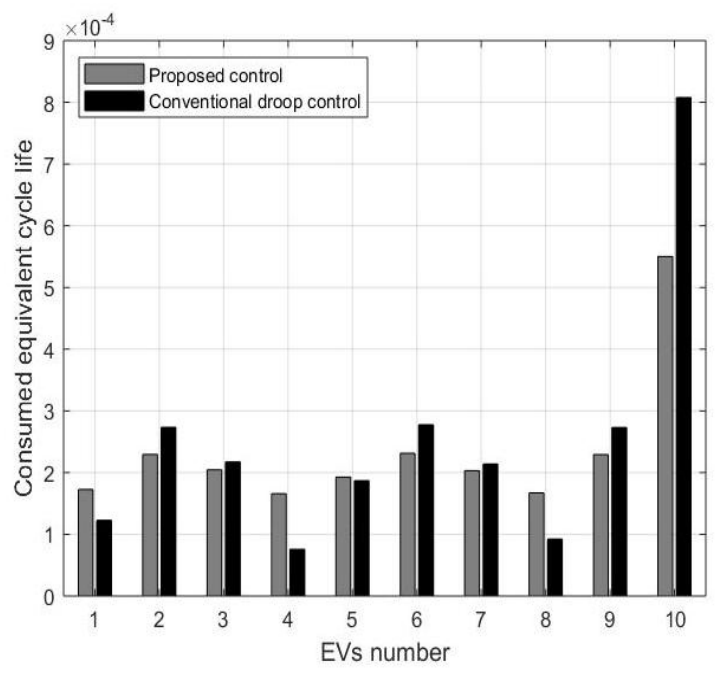

Fig. 5. Consumed equivalent cycle life of EV batteries due to $\mathrm{V} 2 \mathrm{G}$

Table II shows the comparison of the consumed equivalent cycle life of the conventional droop control and the proposed droop control. About $7.7 \%$ of consumed equivalent cycle life for entire EVs which participate in V2G is reduced by applying the proposed droop control for 24 hours V2G operation. And proposed control prevented battery cycle life consumption by V2G operation from being concentrated on some EVs.

Table- II: Consumed equivalent cycle life in case study

\begin{tabular}{|c|c|c|c|}
\hline \multirow{2}{*}{$\begin{array}{c}\text { EV } \\
\text { number }\end{array}$} & \multirow{2}{*}{$\begin{array}{c}\text { Initial } \\
\text { DOD }\end{array}$} & \multicolumn{2}{|c|}{$\begin{array}{c}\text { Consumed equivalent cycle life of } \\
\text { battery for frequency regulation }\end{array}$} \\
\cline { 3 - 4 } & & $\begin{array}{c}\text { Conventional } \\
\text { droop control }\end{array}$ & $\begin{array}{c}\text { Implemented } \\
\text { droop control }\end{array}$ \\
\hline 1 & 0.40497 & 0.00017271 & 0.00012266 \\
\hline 2 & 0.31890 & 0.00022940 & 0.00027340 \\
\hline 3 & 0.44903 & 0.00020451 & 0.00021732 \\
\hline 4 & 0.31091 & 0.00016595 & 0.00007583 \\
\hline 5 & 0.20730 & 0.00019260 & 0.00018701 \\
\hline 6 & 0.32378 & 0.00023137 & 0.00027757 \\
\hline 7 & 0.24463 & 0.00020315 & 0.00021401 \\
\hline 8 & 0.04688 & 0.00016723 & 0.00009236 \\
\hline 9 & 0.31866 & 0.00022930 & 0.00027320 \\
\hline 10 & 0.47513 & 0.00055003 & 0.00080768 \\
\hline Total & & 0.00234625 & 0.00254105 \\
\hline
\end{tabular}

\section{CONCLUSION}

The biggest challenges to realizing V2G service are damage to battery life cycle, owner's inconvenience and EV schedule prediction. If we use $\mathrm{V} 2 \mathrm{G}$ for frequency regulation, it is relatively free from these challenges. However droop control, which normally used for frequency regulation, is not suitable for $\mathrm{V} 2 \mathrm{G}$ frequency regulation. DOD and battery cycle life must be considered in the frequency regulation through $\mathrm{EV}$ to realize the $\mathrm{V} 2 \mathrm{G}$ service. In this paper, we proposed implemented droop control for V2G in frequency regulation. DODs of each EV battery are reflected in droop constant in real time to control the discharge of the battery in proposed method. Proposed control allocates the discharge to prevent from concentrating only on some of the EV batteries. As the discharges are uniformly distributed, cycle life of the EV batteries participating in $\mathrm{V} 2 \mathrm{G}$ are saved and inconvenient of the EV owner is minimized. MATLAB case study shows the effectiveness of proposed control. Compared to conventional droop control, proposed control saves the battery cycle life of the overall $\mathrm{EV}$ by $7.7 \%$. In order to maximize the usability, V2G has to be used both peak shaving and frequency regulation[21]. DOD based V2G control for dual-use will be treated in future work.

\section{ACKNOWLEDGMENT}

This work was supported by National Research Foundation Grant (NRF-2019R1C1C1007566) funded by the Korean government. 


\section{REFERENCES}

1. "Global EV Outlook 2018," International Energy Agency (IEA)2018. https://doi.org/10.1787/9789264302365-en

2. "Electric Vehicle Sales Forecast and the Charging Infrastructure Required Through 2030," Institute for Electric Innovation (IEI)2018.

3. J. A. P. Lopes, F. J. Soares, and P. M. R. Almeida, "Integration of electric vehicles in the electric power system," Proceedings of the IEEE, vol. 99, no. 1, pp. 168-183, 2011 https://doi.org/10.1109/JPROC.2010.2066250

4. V. Silva and C. J. E. Kieny, Germany: RWE Deutschland AG, "Impacts of $\mathrm{EV}$ on power systems and minimal control solutions to mitigate these," 2011.

5. J. C. Gómez and M. M. Morcos, "Impact of EV battery chargers on the power quality of distribution systems," IEEE Transactions on Power Delivery, vol. 18, no. 3, pp. 975-981, 2003. https://doi.org/10.1109/tpwrd.2003.813873

6. J. Taylor, A. Maitra, M. Alexander, D. Brooks, and M. Duvall, "Evaluations of plug-in electric vehicle distribution system impacts," in IEEE PES General Meeting, 2010, pp. 1-6: IEEE.

7. C. Dharmakeerthi, N. Mithulananthan, and T. Saha, "Impact of electric vehicle fast charging on power system voltage stability," International Journal of Electrical Power Energy Systems, vol. 57, pp. 241-249, 2014. https://doi.org/10.1016/j.ijepes.2013.12.005

8. G. Putrus, P. Suwanapingkarl, D. Johnston, E. Bentley, and M. Narayana, "Impact of electric vehicles on power distribution networks," in 2009 IEEE Vehicle Power and Propulsion Conference, 2009, pp. 827-831: IEEE.

9. C. Guille and G. Gross, "A conceptual framework for the vehicle-to-grid (V2G) implementation," Energy policy, vol. 37, no. 11, pp. 4379-4390, 2009. https://doi.org/10.1016/j.enpol.2009.05.053

10. Z. Wang and S. Wang, "Grid power peak shaving and valley filling using vehicle-to-grid systems," IEEE Transactions on power delivery, vol. 28, $\begin{array}{llll}\text { no. } 3, & \text { pp. } & 1822-1829, & \end{array}$ https://doi.org/10.1109/tpwrd.2013.2264497

11. J. Y. Yong, V. K. Ramachandaramurthy, K. M. Tan, and N. Mithulananthan, "A review on the state-of-the-art technologies of electric vehicle, its impacts and prospects," Renewable Sustainable Energy Reviews, vol. 49, pp. 365-385, 2015. https://doi.org/10.1016/j.rser.2015.04.130

12. E. L. Karfopoulos and N. D. Hatziargyriou, "Distributed coordination of electric vehicles providing V2G services," IEEE Transactions on Power Systems, vol. 31, no. 1, pp. 329-338, 2016. https://doi.org/10.1109/tpwrs.2015.2395723

13. A. Y. Saber and G. K. Venayagamoorthy, "Optimization of vehicle-to-grid scheduling in constrained parking lots," in 2009 IEEE Power \& Energy Society General Meeting, 2009, pp. 1-8: IEEE.

14. M. Aziz, T. Oda, T. Mitani, Y. Watanabe, and T. Kashiwagi, "Utilization of electric vehicles and their used batteries for peak-load shifting," Energies, vol. 8, no. 5, pp. 3720-3738, 2015. https://doi.org/10.3390/en8053720

15. I. Duggal and B. Venkatesh, "Short-Term Scheduling of Thermal Generators and Battery Storage With Depth of Discharge-Based Cost Model," IEEE Transactions on Power Systems, vol. 30, no. 4, pp. 2110-2118, 2015. https://doi.org/10.1109/tpwrs.2014.2352333

16. T. Guena and P. Leblanc, "How depth of discharge affects the cycle life of lithium-metal-polymer batteries," in INTELEC 06-Twenty-Eighth International Telecommunications Energy Conference, 2006, pp. 1-8: IEEE.

17. C. Peng, J. Zou, L. Lian, and L. J. A. e. Li, "An optimal dispatching strategy for V2G aggregator participating in supplementary frequency regulation considering EV driving demand and aggregator's benefits," Applied energy, vol. 190, pp. 591-599, 2017 https://doi.org/10.1016/j.apenergy.2016.12.065

18. W. Kempton et al., "A test of vehicle-to-grid (V2G) for energy storage and frequency regulation in the PJM system," Results from an Industry-University Research Partnership, vol. 32, 2008.

19. P. Kundur, N. J. Balu, and M. G. Lauby, Power system stability and control. McGraw-hill New York, 1994.

20. B. M. Weedy, B. J. Cory, N. Jenkins, J. B. Ekanayake, and G. Strbac, Electric power systems. John Wiley \& Sons, 2012.

21. C. D. White and K. M. Zhang, "Using vehicle-to-grid technology for frequency regulation and peak-load reduction," Journal of Power Sources, vol. 196, no. 8, pp. 3972-3980, 2011. https://doi.org/10.1016/j.jpowsour.2010.11.010

\section{AUTHORS PROFILE}

Author-1 Photo

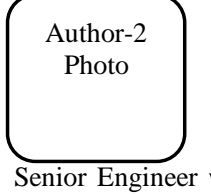

Minhan Yoon received the B.S. degree and the Ph.D. degree from the Department of Electrical Engineering, Korea University, Seoul, Korea, in 2009 and 2015, respectively. He was a Post-doctoral Research Associate in Seoul National University, Seoul, Korea, in 2015, and a ngineer with Korea Electrotechnology Research Institute (KERI) during 2015-2017. In 2017, he joined the Department of Electrical Engineering, Tongmyong University, Busan, Korea, as an Assistant Professor. His research interests include power system analysis considering HVDC, FACTS and AC-DC system interactions.

\section{Author-3}

Photo

Jaewan Suh received a B.S. and Ph.D. degrees from the School of Electrical Engineering, Korea University, in 2011 and 2017. He worked in Korea Institute of Science and Technology Evaluation and Planning as an Associate Research Fellow for one year. He is presently an Assistant Professor of Department of Electrical Engineering at Dongyang Mirae University. His research interests include power quality, power system frequency regulation and distributed generation 